\title{
O Processo Psicoterapêutico de uma Criança: Análise Baseada no Child Psychotherapy Q-Set
}

\author{
Cibele Carvalho ${ }^{1}$ \\ Lucia Rech Godinho \\ Vera Regina Röhnelt Ramires \\ Programa de Pós-Graduação em Psicologia da Universidade do Vale do Rio dos Sinos, \\ São Leopoldo, RS, Brasil
}

\begin{abstract}
Resumo
Este estudo teve como foco a análise do primeiro ano do processo psicoterapêutico de uma criança, com base no Child Psychotherapy Q-set (CPQ). O estudo teve delineamento descritivo, longitudinal, adotando-se o procedimento de estudo de caso sistemático. Participaram um menino de sete anos de idade e sua terapeuta. O CPQ foi aplicado em duas sessões do início do tratamento, duas sessões após três meses, duas sessões após seis meses, duas sessões após nove meses e duas sessões após um ano, para identificar as características do processo psicoterapêutico, ao longo do tempo. Os resultados obtidos revelaram as características dessa psicoterapia, como ela se modificou ao longo do tempo e como essas modificações podem ser indicativas de evolução do processo terapêutico. As atitudes da terapeuta envolveram, inicialmente, um trabalho interpretativo e, posteriormente mais reflexivo e de apoio. A criança, inicialmente, mostrou-se resistente e com poucos recursos internos, mas, ao longo do tratamento, revelou maior abertura para o trabalho terapêutico, abordando conteúdos significativos em relação à sua problemática. Algumas características da interação terapeuta-paciente foram se tornando mais salientes ao longo do tempo.
\end{abstract}

Palavras-chave: Psicoterapia, crianças, pesquisa de processo.

\section{Child Psychotherapeutic Process: Analysis Based on the Child Psychotherapy Q-Set}

\begin{abstract}
This study has focused on the analysis of psychotherapeutic process in the first year of a child, based on the Child Psychotherapy Q-Set (CPQ). The research guided by the longitudinal descriptive approach was made, adopting the Systematic Case Study procedure. Participants were a 7-year-old boy and his therapist. The CPQ was administered in two sessions in beginning the of treatment, two sessions after three months, two sessions after six months, two sessions after nine months and in two sessions after a year, to identify the characteristics of the therapeutic process, over the time. The findings revealed the characteristics of psychotherapy, as it has changed over time and how these changes may be indicative of outcome in therapy. The therapist attitudes involved, initially, an interpretive work and subsequently more reflective and support. The child initially was resistant and with few internal resources, but during
\end{abstract}

1 Endereço para correspondência: Rua Pinheiro Machado, 861, Centro, Lajeado, RS, Brasil 95900-000. Fone: (51) 9857-0912. E-mail: cibele.carvalho8@gmail.com

Os autores agradecem ao Conselho Nacional de Desenvolvimento Científico e Tecnológico (CNPq) pelo financiamento do projeto de pesquisa e à Coordenação de Aperfeiçoamento de Pessoal de Nível Superior (CAPES) pela bolsa de Mestrado da primeira autora. 
treatment, showed greater openness to the therapeutic work, addressing significant content in relation to their problems. The interaction characteristics also became more prominent over time.

Keywords: Psychotherapy, children, process research.

\section{EI Proceso Psicoterapéutico de un Niño: Un Análisis Basado en Child Psychotherapy Q-Set}

\section{Resumen}

Este estudio se centró en el análisis del primer año del proceso psicoterapéutico de un niño, basado en la psicoterapia infantil $Q$-set (CPQ). El estudio fue el diseño descriptivo, longitudinal, la adopción de un procedimiento sistemático estudio de caso. Participó en un niño de siete años de edad, y su terapeuta. El CPQ se aplicó en dos sesiones que inician el tratamiento, dos sesiones después de tres meses, dos sesiones después de seis meses, dos sesiones después de nueve meses y dos sesiones después de un año, para identificar las características del proceso psicoterapéutico, a través del tiempo. Los resultados revelaron las características de la psicoterapia, ya que ha cambiado con el tiempo y cómo estos cambios pueden ser indicativos de los resultados en la terapia. Las actitudes de terapeutas involucrados, en un principio, un trabajo interpretativo y, posteriormente, más reflexivo y apoyo. El niño inicialmente era resistente y con pocos recursos internos, pero durante el tratamiento, mostró una mayor apertura al trabajo terapéutico, abordando contenidos significativos en relación con sus problemas. Algunas características de la interacción paciente-terapeuta hicieron más prominentes con el tiempo.

Palabras clave: Psicoterapia, niños, investigación processo.

O foco deste estudo foi a análise do processo do primeiro ano de psicoterapia psicanalítica de uma criança. Essa temática vem ao encontro da necessidade de pesquisas empíricas que demonstrem de que forma ocorre a efetividade do tratamento psicanalítico com crianças, com o intuito de aprimorar a prática clínica e, desta forma, mantê-la em sintonia com as demandas contemporâneas da população (Midgley \& Kennedy, 2011).

A psicoterapia psicanalítica tem o objetivo de possibilitar ao indivíduo a ampliação do entendimento sobre seu funcionamento, resultando no uso de defesas mais maduras, na melhora do padrão das relações objetais e em mudanças sintomáticas significativas (Eizirik \& Hauck, 2007). Glenn (1996) postulou que o aspecto essencial do tratamento psicanalítico com crianças é a interpretação das pulsões e defesas conflitantes do paciente, permitindo que o ego passe a funcionar de modo mais adaptativo, possibilitando a alteração das defesas, a modificação do superego e uma maior gratificação pulsional. Espera-se que, ao longo do tratamento, as tendências regres- sivas da criança diminuam, haja superação das inibições e das paradas desenvolvimentais e que as energias liberadas e neutralizadas fiquem disponíveis para a atividade sublimada.

Para alcançar esses objetivos, Castro, Campezatto e Saraiva (2009) dividem os objetivos do processo terapêutico com crianças em três fases: inicial, intermediária e final. O início do tratamento é caracterizado pela construção de um vínculo de confiança e da aliança de trabalho entre a criança e o terapeuta. Almeja-se que, nessa etapa, a criança reduza os sentimentos persecutórios, familiarize-se com o seeting e o processo terapêutico e, com isso, alie-se, cada vez mais, ao terapeuta, na tarefa de identificar conflitos e buscar elaborá-los.

A fase intermediária do tratamento pode ser caracterizada por uma maior familiaridade entre terapeuta e paciente. Visa analisar, explorar e resolver sintomas e as dificuldades emocionais do paciente. A aliança terapêutica já estabelecida possibilita que a criança comece a expressar desagrado, raiva ou aversão ao terapeuta ou a externalizar seus impulsos durante a sessão (Cal- 
berg, 2009). O terapeuta procura mostrar para a criança e, eventualmente, interpretar, suas atitudes ou comportamentos, ou estimula a criança a falar de seus problemas. Um aspecto importante do papel do terapeuta é ajudar o paciente a se tornar consciente de padrões inconscientes expressados na sua conduta não verbal, para que, ao final, possa ganhar um senso de domínio e entendimento do que está sendo repetido num relacionamento após outro (Gabbard \& Westen, 2003). Na medida em que a psicoterapia evolui, pode-se esperar que a criança alcance insights cada vez mais genuínos e significativos, com consequente alívio dos sintomas e com crescimento mental, brincando de forma mais simbólica, diminuindo o uso de mecanismos regressivos e de actings (Zavaschi, Bassols, Bergmann, \& Costa, 2005).

A fase final do tratamento caracteriza-se por uma criança com uma noção de self integrado, sentimentos de segurança, menos uso de mecanismos de defesa primitivos, reconhecendo suas necessidades e desejos, adquirindo a capacidade de auto-reflexão e autoanálise (Luyten, Blatt, \& Mayes, 2012). Espera-se que, nesta etapa, a criança tenha elaborado seus conflitos manifestos e latentes que originaram a busca do tratamento (Castro et al., 2009).

Atualmente, há uma discussão, no campo das psicoterapias, de como a pesquisa pode evidenciar o que acontece quando um adulto e uma criança se encontram, em um tratamento psicoterápico. Compreender por que e como as mudanças acontecem tem o potencial de auxiliar a identificar os ingredientes ativos ou mecanismos de mudança que estão na base de uma intervenção clínica bem sucedida (Midgley, 2007; Schneider, Midgley, \& Duncan, 2010). Esse é o melhor investimento, tanto a curto como em longo prazo, para aprimorar a prática clínica e o cuidado com os pacientes (Kazdin \& Nock, 2003).

\section{Pesquisas em Psicoterapia Psicanalítica de Crianças}

Nos últimos anos, tem havido progressos consideráveis na pesquisa em psicoterapia de crianças e adolescentes, refletida na quantidade e qualidade de estudos de resultados e a identificação de tratamentos baseados em evidências para uma série de problemas clínicos (Midgley, Anderson, Grainger, Vuckovic-Nesic, \& Urwin, 2009; Palmer, Nascimento, \& Fonagy, 2013). Esse avanço também tem ocorrido no campo das pesquisas em psicoterapia psicanalítica e alguns resultados importantes têm sido demonstrados. Por exemplo, o fato de que algumas crianças parecem ser mais responsivas que outras, indicando que as mais jovens (entre seis e nove anos) se beneficiam mais do tratamento do que as mais velhas (entre nove e doze anos; Deakin \& Nunes, 2009; Midgley \& Kennedy, 2011) e que o trabalho com os pais ou com a família se constitui como um importante componente do tratamento dessa população (Midgley \& Kennedy, 2011; Odhammar, Sundin, Jonson, \& Calberg, 2011). Além disso, diversas pesquisas mostram que as melhoras atingidas com o tratamento foram mantidas ou se intensificaram nas avaliações de follow-up (Midgley \& Kennedy, 2011; Odhammar et al., 2011).

No entanto, apesar dos avanços obtidos, nas pesquisas de resultado, questões fundamentais, sobre os tratamentos e seus efeitos, permanecem (Kazdin \& Nock, 2003). A maioria dos estudos revela a ausência de considerações de como ou por que alguns tratamentos funcionam e quais aspectos do tratamento tendem a facilitar ou inibir a mudança terapêutica.

Conforme Oliveira (2012), os desafios para a pesquisa em psicoterapia psicanalítica com crianças são, ainda, muito grandes, pois essa forma de tratamento traz dificuldades adicionais ao pesquisador e ao clínico, que se defronta com um número ilimitado de moderadores de tratamento, que podem influenciar o resultado. Exemplos incluem as características da criança (idade no início da psicoterapia, gravidade da psicopatologia, comorbidade), dos pais (presença de psicopatologia, estresse), do terapeuta (experiência, características de personalidade), da família (dinâmica familiar, relacionamentos, discórdia), e contexto social (escola, vizinhança, cultura).

Há um reconhecimento crescente de que apenas a exploração cuidadosa da interação do paciente com o terapeuta pode ilustrar os aspectos centrais do tratamento psicanalítico e permitir uma teoria empiricamente orientada 
do processo. Assim, os extensos esforços de pesquisa sobre o processo terapêutico que começam a elucidar, por exemplo, a contribuição do terapeuta, incluindo sua personalidade e técnicas, são cruciais (Strupp, 2013). A avaliação do processo terapêutico centra-se tanto na interação terapêutica como no processo de mudança experimentado durante a relação terapêutica, estudando os episódios relevantes de mudança (Brum et al., 2012).

Em 2007, Midgley, revisou 15 estudos que abordavam algum aspecto do processo psicoterápico com crianças, tais como o comportamento da criança no setting clínico, o brinquedo, o funcionamento defensivo, a qualidade das relações de objetos, os padrões de transferência, as interações entre o terapeuta e a criança, a aliança terapêutica e os turning points na psicoterapia. $\mathrm{O}$ autor apontou para a necessidade de capturar integralmente o encontro terapêutico, em toda a sua complexidade, superando o foco em aspectos específicos do processo.

No contexto brasileiro, Deakin e Nunes (2008) identificaram apenas nove estudos publicados entre 1995 e 2005 destinados a pesquisar a psicoterapia infantil. A ênfase das pesquisas foi nos resultados de psicoterapias comportamentais, havendo uma parcela mínima de estudos em psicoterapias psicanalíticas e/ou psicodinâmicas. As autoras apontaram para a dificuldade de adequar métodos rígidos e controlados a um processo subjetivo como o proposto pela psicanálise, o que sinaliza a importância de buscar métodos próprios de investigação para esta abordagem terapêutica.

\section{Avaliação do Processo em Psicoterapia de Crianças: O Child Psychotherapy Q-Set}

A viabilidade e a qualidade dos estudos sobre o processo de psicoterapia requerem instrumentos e procedimentos que permitam capturá-lo da forma mais acurada possível. Tendo em vista essa necessidade, foi desenvolvido o Child Psychotherapy Q-Set (CPQ; Schneider \& Jones, 2006, 2012).

O CPQ foi inspirado no Psychotherapy Process Q-Set (PQS), elaborado por Jones
(1988, 2000) com base na Q-metodologia, em uma tentativa de obter uma medida empírica do processo da psicoterapia de adultos. O instrumento é composto por 100 itens que descrevem aspectos que podem ser mais ou menos salientes numa sessão de psicoterapia. Uma distribuição forçada é feita, em curva normal, em nove categorias, que identificam desde os itens mais característicos da sessão aos menos característicos. O PQS foi reconhecido pela sua efetividade em capturar a complexidade do processo terapêutico com adultos e tem contribuído para informar sobre a relação do processo com o resultado do tratamento.

Para o desenvolvimento do CPQ, Schneider (2004), partindo de uma revisão da literatura e de pesquisas em psicoterapia com crianças, reuniu 100 itens característicos de processos que ocorrem no tratamento com essa população, de acordo com orientações teóricas distintas. Os itens se dividem em três categorias: (a) experiência, comportamento e atitudes do paciente; (b) comportamentos e atitudes do terapeuta e (c) a interação terapeuta-paciente.

O processo da psicoterapia é caracterizado pela estabilidade ou variabilidade de itens, uns em relação aos outros durante uma sessão, formando um perfil de cada uma. Estes perfis podem ser estudados no curso do tratamento (Jones, 2000; Schneider \& Jones, 2006, 2012). Embora seja aplicável a vários casos de investigação, a técnica $Q$-sort é especialmente qualificada para analisar um único caso em que o $N$ do estudo é o número de sessões de tratamento e não o número de participantes.

No que tange a validade do CPQ, Schneider, Pruetzel-Thomas e Midgley (2009) demonstraram sua validade discriminante ao comparar sessões entre duas modalidades de psicoterapia, a psicodinâmica e a cognitivo comportamental. Diversos estudos, também, vêm confirmando a fidedignidade interavaliadores obtida com o CPQ (Goodman \& Athey-Lloyd, 2011; Schneider, 2003; Schneider et al., 2010). Todos eles têm atingido índices de concordância, entre avaliadores treinados, superiores a 0,70 , em análises independentes de grupos de sessões de psicoterapia de crianças (variando de 20 a 53 sessões). 
Os autores revisados indicam que há um investimento crescente nos estudos de processo da psicoterapia psicodinâmica, e em menor medida com crianças, que vem oferecendo achados importantes para a comunidade científica e para os clínicos dedicados ao trabalho com essa população. No entanto, há a necessidade de que mais estudos sejam desenvolvidos, utilizando instrumentos que se propõem a avaliar o processo psicoterápico com crianças, pois, como se pode constatar, os estudos com este foco, ainda são em número reduzido e o processo psicoterapêutico é, ainda, pobremente compreendido. Fica evidente, assim, a importância de direcionar esforços de pesquisas para o processo da psicoterapia com essa população, compreendendo seus mecanismos de mudança e que fatores contribuem para uma intervenção mais ou menos bem sucedida. Com base nessas premissas, esse estudo teve como objetivo descrever e analisar as principais características do processo do primeiro ano de psicoterapia psicanalítica de uma criança, de acordo com o CPQ.

\section{Método}

\section{Participantes}

Valter $^{2}$, sete anos de idade no início do atendimento, filho único, cursava o primeiro ano do ensino fundamental. Os pais, profissionais de classe média, buscaram ajuda devido às dificuldades de relacionamentos do menino, na escola e, também, por demonstrar alguns sinais de ansiedade. O menino apresentava excesso de peso.

Nas entrevistas iniciais, os pais referiram que Valter não tinha amigos na escola, que as crianças o chamavam de "chorão", "bebezão" e faziam "musiquinhas debochando dele". Valter tinha dificuldade de dividir os amigos e pedia exclusividade nas relações com os colegas. A mãe relatou, também, que, quando ficava com raiva, o menino perdia o controle, como na situação em que brigou na escola, empurrou a classe sobre um colega e pegou o menino pelo pescoço.

2 O nome do paciente, assim como qualquer dado que pudesse identificá-lo, foi modificado.
Porém, até o ano anterior, Valter apanhava dos colegas na escola e teve que mudar de turma por isto. Em casa, às vezes, dizia que queria ficar sozinho. Dormia no quarto dos pais.

Sua psicoterapeuta tinha 23 anos de experiência clínica, é especialista em psicoterapia psicanalítica e Mestre em Psicologia Clínica. Foi convidada a participar do estudo, por conveniência.

Os critérios de inclusão dos participantes no estudo foram a criança estar nos primeiros anos do Ensino Fundamental, apresentar necessidade e desejo de realizar a psicoterapia, contar com a concordância dos pais em relação à psicoterapia e à inserção na pesquisa e a terapeuta possuir formação e experiência clínica em psicoterapia psicanalítica com crianças. A terapeuta, além da ciência e concordância com a pesquisa, não tinha conhecimento acerca do projeto de pesquisa, da análise das sessões ou qualquer outra informação ou interação com o grupo de pesquisa.

\section{Delineamento}

Foi realizado um estudo descritivo, longitudinal, caracterizado como um estudo de processo e que adotou o procedimento de Estudo de Caso Sistemático - ECS (Edwards, 2007). O ECS é um tipo específico de pesquisa de caso único, cujo objetivo é a compreensão dos fatores que contribuem para a mudança no processo psicoterápico. Caracteriza-se por ser um estudo idiográfico, longitudinal e intensivo, que constitui uma extensão da prática clínica e lança mão de diversos mecanismos em busca do rigor metodológico e do controle dos vieses do estudo. O ECS está baseado em procedimentos desenvolvidos no contexto clínico ou naturalista e pressupõe avaliações de variáveis do paciente, do terapeuta e da interação entre eles (Eells, 2007).

O interesse pelo delineamento de Estudo de Caso tem aumentado, nos últimos anos, demonstrando uma necessidade de aproximação da pesquisa com a prática clínica (Kazdin \& Nock, 2003; Midgley et al., 2009). Nesse sentido, há o reconhecimento crescente de que os Estudos Controlados Randomizados não oferecem elementos acerca de que forma os pacientes 
mudam. Os estudos naturalísticos, que ocorrem no setting clínico usual, oferecem uma oportunidade para compreender os processos que promovem mudança terapêutica, o que requer uma análise mais próxima e mais profunda das interações terapeuta-paciente (Goodman, Anderson, \& Diener, 2014; Jones, 2000).

\section{Instrumento}

Foi utilizado o CPQ (Schneider \& Jones, 2006, 2012) cujo objetivo é proporcionar um procedimento de avaliação padrão para a descrição e análise do processo psicoterapêutico. Os 100 itens, utilizados para a codificação das sessões, são impressos em cartões individuais. Para permitir maior confiabilidade, um manual de codificação oferece definições claras e exemplos de cada item, refletindo características que podem ser identificadas usando videotapes das sessões. O instrumento foi traduzido e adaptado para o Português brasileiro por Ramires e Schneider (no prelo) e seguiu procedimento similar ao realizado com o PQS (Serralta, Nunes, \& Eizirik, 2007). Na primeira etapa, duas traduções independentes do manual e dos itens do instrumento foram realizadas por duas psicólogas bilíngues. A seguir, com o auxílio de uma terceira psicóloga, também bilíngüe, foi desenvolvida a versão consensual, levando em consideração as duas versões preliminares. Em alguns itens, optou-se por uma ou outra versão e, em outros, por uma conjugação de ambas. A segunda etapa foi a retrotradução da versão consensual para o idioma do original, o inglês. Essa etapa foi realizada por duas tradutoras bilíngües, experientes em tradução e retrotradução de instrumentos de pesquisa cuja língua nativa é o português. A terceira etapa foi a avaliação da equivalência semântica feita por duas psicólogas bilíngues. A avaliação levou em consideração a concordância das ideias do instrumento original com a versão traduzida. A versão retrotraduzida foi enviada para a autora do instrumento original e foi aprovada. A quarta etapa consistiu em utilizar o instrumento no treinamento dos juízes que atuariam como juízes no caso em questão. Obteve-se índice de concordância entre avaliadores maiores que 0,7 , critério adotado em outros trabalhos com o CPQ (Goodman \& Athey-Lloyd, 2011; Schneider, 2003; Schneider et al., 2010).

\section{Procedimentos de Coleta de Dados}

A partir da busca por tratamento pelos pais da criança, primeiramente, foi realizada a avaliação do caso, para confirmar a necessidade e o desejo de realizar a psicoterapia. Os instrumentos utilizados nesta etapa foram entrevistas com pais para análise do motivo da consulta, levantamento da anamnese e entrevistas com a criança na modalidade de Hora de Jogo (Efron, Fainberg, Kleiner, Sigal, \& Woscoboinik, 2003), que identificaram indicadores de ansiedade e depressão associados às dificuldades relacionais do menino. Além disso, a criança mostrou poucos recursos psíquicos e pouca capacidade de simbolização.

Nesse momento, também, foi apresentada e esclarecida a proposta da pesquisa, explicitando a filmagem das sessões da criança, durante todo o tratamento, para os pais e para a criança, consultando-os sobre a possibilidade e o desejo de participação. Com a concordância de todos, o tratamento foi iniciado.

A câmera filmadora (equipamento bastante pequeno e discreto) era colocada no consultório pela própria terapeuta, ao iniciar o atendimento, e desligada ao finalizá-lo. Caso criança ou terapeuta se sentissem constrangidos com a filmagem, a pesquisa poderia ser interrompida visando, em primeiro lugar, o bem estar da criança e a prioridade do seu melhor atendimento. A psicoterapia foi conduzida a partir do enfoque psicanalítico, como mencionado anteriormente (Eizirik \& Hauck, 2007; Glenn, 1996).

O tratamento foi realizado em um consultório de psicologia, devidamente equipado para a realização da atividade de psicoterapia. As sessões da criança foram semanais, com duração de 50 minutos cada uma. Periodicamente, foram realizadas entrevistas de acompanhamento com os pais, com a mesma terapeuta, com o objetivo de coletar informações adicionais sobre a criança e orientá-los em relação ao processo de psicoterapia. 
O estudo foi aprovado pelo Comitê de Ética em Pesquisa da universidade a qual se vinculam as pesquisadoras. Todos os cuidados éticos foram respeitados, priorizando-se o bem-estar dos participantes. Através do Termo de Consentimento Livre e Esclarecido, garantiu-se ao paciente e seus responsáveis, assim como à terapeuta, o direito de interromper sua participação em qualquer momento, sem prejuízo da psicoterapia em andamento e, também, de ser encaminhado a outro serviço de saúde, se necessário.

\section{Procedimentos de Análise dos Dados}

Para análise das sessões, quatro juízes, psicólogos com experiência clínica (um aluno de pós-doutorado, um doutorando e dois mestrandos), foram treinados. A coordenadora do projeto mais amplo, ao qual este estudo se vincula, foi treinada pela pesquisadora que desenvolveu o instrumento e por dois pesquisadores que vem trabalhando com o CPQ, na Inglaterra e nos Estados Unidos. Tendo obtido a certificação necessária, foi responsável pelo treinamento dos juízes no Brasil. O treinamento consistiu, primeiramente, na discussão minuciosa do procedimento e sua forma de avaliação das sessões. Posteriormente, dez sessões, de outros casos, foram distribuídas entre os avaliadores recém-treinados, sorteados em duplas, para que realizassem o ordenamento dos 100 itens do CPQ, de modo independente. $\mathrm{O}$ treinamento buscou alcançar um alto grau de concordância $(0,70)$ entre as pontuações independentes de cada juiz e só foi finalizado quando essa pontuação foi alcançada. Sempre que a concordância era menor que 0,7 , as discordâncias eram discutidas e a sessão avaliada era descartada, sendo realizada a avaliação de nova sessão.

Das 52 sessões realizadas no primeiro ano de tratamento, foram selecionadas dez para serem analisadas: as duas primeiras sessões da psicoterapia, duas sessões de quando a psicoterapia completou três meses, duas sessões aos seis meses, duas sessões aos nove meses e duas sessões do final do primeiro ano. Cada sessão foi analisada por dois juízes independentes, formando pares aleatórios e que se intercambiavam. Os juízes eram cegos quanto ao número da sessão, ao resultado do tratamento, e à identidade e as avaliações dos outros juízes.

Após assistirem ao vídeo da sessão terapêutica, os avaliadores ordenaram os cartões (itens) em nove pilhas dispostas num continuum que vai desde os itens menos característicos (categoria 1) até os mais característicos (categoria 9). O número de cartões em cada pilha é pré determinado a fim de se obter uma distribuição normal. Cinco cartões são colocados nas categorias $1 \mathrm{e}$ 9. Dezoito cartões são colocados na categoria do meio (catogoria 5). O número de cartões aumenta entre as categorias 1 e 5 e diminui entre as categorias 5 e 9 . O ordenamento Q utiliza escala ipsativa em que os itens são avaliados uns em relação aos outros ao invés de comparados a algum parâmetro objetivo, como é o caso das escalas normativas (Jones, Hall, \& Parke, 1991).

O número da pilha em que, ao final do ordenamento, cada cartão foi colocado é registrado e reflete o quanto esse item caracteriza o processo terapêutico em comparação com os outros itens. As sessões receberam um escore final resultante da média das classificações pelos dois juízes, mesmo procedimento utilizado em estudos prévios (Goodman \& Athey-Lloyd, 2011; Schneider et al., 2010). Foram realizadas análises estatísticas para verificação da concordância entre os avaliadores através do índice de correlação intraclasse, o coeficiente alpha de Cronbach. A confiabilidade interavaliadores, em todas as sessões, variou entre 0,70 e 0,81 (média 0,75 ). Sempre quando a confiança entre qualquer um dos pares de juízes ficou abaixo de 0,70 , um juiz adicional classificou a sessão. Posteriormente, as médias das avaliações do CPQ foram calculadas para gerar o escore composto que foi utilizado nas análises subsequentes.

Após essa análise inicial, para obter um resumo quantitativo do processo terapêutico, foi calculada e ordenada a média dos itens do CPQ nas sessões. Com isso foram identificados os itens mais e menos característicos de cada uma, como também a média dos itens mais e menos característicos das dez sessões avaliadas ao longo do primeiro ano do tratamento. Estas análises foram realizadas utilizando o software SPSS 21.0. 


\section{Resultados}

No processo psicoterápico, Valter evidenciou, inicialmente, muita dificuldade para abordar suas dificuldades de relacionamento, como também para pensar sobre suas emoções e sentimentos relacionados às situações conflitivas, reagindo de maneira muito regressiva. Ao longo do primeiro ano de tratamento, tornou-se menos impulsivo e mais tolerante às intervenções terapêuticas. Além disso, passou a expressar seus sentimentos, principalmente os negativos, evidenciando seus conflitos centrais.

A Tabela 1 destaca os itens mais e menos característicos das dez sessões de psicoterapia, ao longo do primeiro ano de tratamento, de acordo com o CPQ.

\section{Tabela 1}

Dez Itens Mais e Menos Característicos no Primeiro Ano de Psicoterapia

Itens do terapeuta

(31) T solicita mais informação ou elaboração

(3) Os comentários de T visam encorajara fala de C

(36) T assinala o uso de defesas por parte de C

(2) T comenta sobre o comportamento não verbal de C (ex. postura corporal, gestos)

(50) T chama a atenção para sentimentos considerados inaceitáveis por C (ex. raiva, inveja ou excitação)

(6) T é sensível aos sentimentos de C

Itens da criança

(58) C parece relutante em examinar pensamentos, reações ou motivações relacionados aos problemas

(42) C ignora ou rejeita os comentários e observações de T

(84) C expressa raiva ou sentimentos agressivos

Itens da interação

(45) $\mathrm{T}$ tolera os fortes afetos ou impulsos de C

Itens menos característicos

Itens do terapeuta

(9) T não é responsivo (vs. afetivamente engajado)

(89) $\mathrm{T}$ age para fortalecer as defesas existentes

(17) T ativamente exerce controle sobre a interação (ex. estruturando, introduzindo novos tópicos)

(55) T recompensa diretamente comportamentos desejáveis

(57) T tenta modificar distorções nas crenças de C

Itens da criança

(53) C transmite consciência das próprias dificuldades internas

(78) C é complacente

(40) C se comunica sem afeto

(32) C alcança uma nova compreensão ou insight

(5) $\mathrm{C}$ tem dificuldade para compreender os comentários de T 
Por meio da análise dos itens mais e menos característicos do processo terapêutico, no período do primeiro ano, pode-se observar que a terapeuta evidenciou uma postura analítica, chamando atenção para sentimentos considerados inaceitáveis pela criança, assinalando o uso de defesas e comentando seu comportamento não verbal. Aliado a isso, manteve uma postura em- pática e sensível aos sentimentos do paciente. Valter apresentou dificuldades para usufruir do trabalho terapêutico, demonstrando resistência ou limitação de recursos internos para analisar pensamentos, reações ou motivações relacionadas aos problemas, não transmitindo consciência das próprias dificuldades internas. Em relação à interação, observou-se que a terapeuta foi tolerante aos fortes afetos e impulsos da criança.

Tabela 2

Itens Mais Característicos dos Cinco Períodos Avaliados no Primeiro Ano de Psicoterapia

Itens do CPQ

Média Média Média Média Média

tempo tempo tempo tempo tempo

$\begin{array}{lllll}1 & 2 & 3 & 4 & 5\end{array}$

Itens do terapeuta

(2) T comenta sobre o comportamento não verbal de C (ex. postura corporal, gestos)

(3) Os comentários de $\mathrm{T}$ visam encorajara fala de $\mathrm{C}$

(6) T é sensível aos sentimentos da criança

$\begin{array}{ccccc}- & 8,5 & 8,5 & 7,5 & 8,25 \\ 9 & 8,25 & 7,5 & - & 7,5 \\ 7,75 & - & 7,5 & - & 8 \\ 8,75 & 8 & 7,5 & 7,25 & 7,25 \\ - & 9 & 8,75 & 8,25 & - \\ 8,25 & - & 8 & - & -\end{array}$

(46) $\mathrm{T}$ interpreta o significado do jogo de $\mathrm{C}$

(50) T chama a atenção para sentimentos considerados inaceitáveis pela criança (ex. raiva, inveja ou excitação)

(62) T assinala um tema recorrente na experiência ou conduta de C

(67) $\mathrm{T}$ interpreta os desejos, sentimentos ou ideias rejeitados ou inconscientes

(65) T clarifica, reafirma ou reformula comunicações de C

(77) A interação de T com C é sensível ao seu nível de desenvolvimento

(79) $\mathrm{T}$ comenta as mudanças no humor ou nas emoções de $\mathrm{C}$

(86) T é confiante, seguro de si

(97) T enfatiza a verbalização dos estados internos e afetos Itens da criança

(1) C expressa sentimentos negativos (ex. crítica, hostilidade) em direção a T

(20) C é provocadora; desafia T ou as regras e limites da sessão

(42) C ignora ou rejeita os comentários e observações de T

(58) C parece relutante em examinar pensamentos, reações ou motivações relacionadas aos problemas

(71) C se engaja no jogo de faz-de-conta

(84) $\mathrm{C}$ expressa raiva ou sentimentos agressivos Itens da interação

(23) A sessão terapêutica tem um tema ou foco específico

(45) $\mathrm{T}$ tolera os fortes afetos ou impulsos de C

(88) O material da sessão é significativo e relevante em relação aos conflitos de $\mathrm{C}$

\begin{tabular}{|c|c|c|c|c|}
\hline- & 8,5 & 7,75 & 8,75 & - \\
\hline- & 8 & - & - & - \\
\hline- & - & 7,5 & - & - \\
\hline 7,75 & - & - & - & - \\
\hline 7,5 & - & - & - & - \\
\hline- & 7,5 & - & - & - \\
\hline 7,5 & - & - & - & 7,5 \\
\hline 8 & 8 & - & - & - \\
\hline- & - & - & 8 & - \\
\hline- & - & - & 7,5 & - \\
\hline- & - & - & 8,25 & 7,5 \\
\hline 8,5 & 8,75 & 8,5 & 9 & 8,5 \\
\hline 8,5 & - & - & - & - \\
\hline- & 8,25 & - & 8,5 & - \\
\hline- & - & - & - & 8 \\
\hline- & - & 7,5 & 8,25 & - \\
\hline- & - & - & - & 7,25 \\
\hline- & - & - & - & 7,25 \\
\hline
\end{tabular}

(98) A relação terapêutica é discutida 


\section{Análise dos Cinco Períodos Avaliados ao Longo do Primeiro Ano de Tratamento}

A análise da variação dos itens ao longo do tempo permite observar diferentes aspectos do processo terapêutico. Mudanças no comportamento da criança, da terapeuta e na interação de ambos puderam ser observadas. As Tabelas 2 e 3 mostram as características principais da psicoterapia, nos cinco períodos avaliados.

Tabela 3

Itens Menos Característicos dos Cinco Períodos Avaliados no Primeiro Ano de Psicoterapia

\begin{tabular}{|c|c|c|c|c|c|}
\hline Itens do CPQ & $\begin{array}{c}\text { Média } \\
\text { tempo } \\
1\end{array}$ & $\begin{array}{c}\text { Média } \\
\text { tempo } \\
2\end{array}$ & $\begin{array}{c}\text { Média } \\
\text { tempo } \\
3\end{array}$ & $\begin{array}{c}\text { Média } \\
\text { tempo } \\
4\end{array}$ & $\begin{array}{c}\text { Média } \\
\text { tempo } \\
5\end{array}$ \\
\hline \multicolumn{6}{|l|}{ Itens do terapeuta } \\
\hline (9) T não é responsivo & 1 & 1 & 1,25 & 1 & 1 \\
\hline $\begin{array}{l}\text { (17) T ativamente exerce controle sobre a interação (ex. } \\
\text { estruturando, introduzindo novos tópicos) }\end{array}$ & 2,25 & 2 & 3 & 2,75 & 2 \\
\hline (18) T é crítico e transmite falta de aceitação & 2,5 & 2,5 & 2,5 & 2,25 & 3 \\
\hline (57) $\mathrm{T}$ tenta modificar distorções nas crenças de $\mathrm{C}$ & 2,25 & 2,25 & - & - & 2,25 \\
\hline (89) $\mathrm{T}$ age para fortalecer as defesas existentes & 2,25 & 2,5 & - & - & 2 \\
\hline \multicolumn{6}{|l|}{ Itens da criança } \\
\hline (5) C tem dificuldade para compreender os comentários de T & - & - & 3 & 2,75 & 2,25 \\
\hline (32) C alcança uma nova compreensão ou insight & - & - & 2,25 & 2 & - \\
\hline (40) $\mathrm{C}$ se comunica sem afeto & 2 & 1,75 & - & - & 2,25 \\
\hline (44) C se sente cautelosa ou desconfiada & - & - & 2,5 & 2,25 & - \\
\hline (53) C transmite consciência das próprias dificuldades internas & 1,5 & 1,75 & 1,75 & 2 & 2,75 \\
\hline (61) C se sente tímida e envergonhada & 2,25 & 2,25 & - & - & - \\
\hline (78) C é complacente & 2,5 & 2,5 & 2,25 & 2 & 2,75 \\
\hline (95) $\mathrm{O}$ brincar de $\mathrm{C}$ carece de espontaneidade & 2 & 1,75 & 2,75 & 2,75 & - \\
\hline \multicolumn{6}{|l|}{ Itens da interação } \\
\hline (41) C não se sente compreendida pelo terapeuta & - & - & 2,5 & 2,25 & 2,25 \\
\hline
\end{tabular}

Nota. T: Terapeuta, C: Criança.

No início do tratamento (tempo um - primeira e segunda sessão) as atitudes da terapeuta se caracterizaram por uma postura exploratória, empática, não diretiva e sensível aos sentimentos da criança. Nesse período, Valter engajou-se no jogo de faz de conta, brincando de forma espontânea, desinibida e segura e comunicando-se afetivamente. No entanto, demonstrou relutância em examinar pensamentos, reações ou motivações relacionadas aos problemas e não transmitiu consciência das próprias dificuldades internas, não alcançando novas compreensões ou insights. Respostas da criança, como "não sei", "não quero falar sobre isso" eram comuns quando o assunto era a escola e sua dificuldade de relacionamento. Nenhum item que compreende a interação entre terapeuta e paciente foi característico desta etapa.

Nos tempos dois, três e quatro (da $10^{\mathrm{a}}$ sessão à $31^{\text {a }}$ ) a terapeuta mostrou-se mais ativa no setting, evidenciando um aumento do trabalho interpretativo, dando ênfase aos sentimentos considerados inaceitáveis pela criança, principalmente a raiva. $\mathrm{O}$ assinalamento do uso de defesas também esteve presente. Valter expressou sentimentos agressivos e uma relutância para 
examinar pensamentos, sentimentos e motivações. Além disso, continuou não transmitindo consciências das suas dificuldades, nem alcançando novos insights. O brincar, nesse período, girava em torno de guerras e batalhas entre monstros e soldados, no entanto, qualquer apontamento, por parte da terapeuta, sobre o estado mental de Valter ou dos bonecos, como "acho que ele está brabo" ou "acho que está com medo" era respondido, prontamente, com "não está sentindo nada", "fica quieta", "eu não quero falar sobre isso".

No tempo quatro $\left(30^{\mathrm{a}}\right.$ e $\left.31^{\mathrm{a}}\right)$, o menino adotou uma postura mais hostil e provocadora em relação à terapeuta, ignorando seus comentários e observações. Nessas sessões, Valter passou boa parte do tempo sentado na poltrona, lendo um livro ou revista, sem interagir com a terapeuta. Respondia a qualquer comentário da terapeuta com "fica quieta", "eu não quero falar", "me deixa ler", o que envolveu uma combinação de trabalho interpretativo e empatia, por parte dela. A interação, neste período, foi caracterizada pela permissão e tolerância da terapeuta da expressão dos fortes afetos e impulsos da criança e pela sensação, transmitida por Valter, de que era compreendido.

No tempo cinco ( $40^{\mathrm{a}}$ e $41^{\mathrm{a}}$ sessão) pode-se verificar uma relevante mudança na caracterização do processo terapêutico. Houve um aprofundamento da análise de temas conflituosos, principalmente relacionados ao déficit relacional de Valter, conforme exemplificado pelos itens do CPQ mais característicos nesse período (23 - a sessão terapêutica tem um tema ou foco específico, 88 - o material da sessão é significativo e relevante em relação aos conflitos da criança, e 98 - a relação terapêutica é discutida). Esses itens correspondem à interação da criança com a terapeuta. Na sessão 40, Valter chega perto da terapeuta e fala: "Quero te falar um coisa, tenho problemas na escola". Em outro momento, diz: "quero te contar uma coisa, eu tenho medo de escuro... e de altura, também". Nesse momento pareceu haver uma abertura deste ao processo psicoterápico, mostrando-se mais disponível e envolvido emocionalmente, na medida em que a hostilidade e a atitude provocadora desaparece- ram nesse período $(1-\mathrm{C}$ expressa sentimentos negativos em direção a $\mathrm{T}$ e $20-\mathrm{C}$ é provocadora; desafia $\mathrm{T}$ ou as regras e limites da sessão). No entanto, ainda demonstrava resistência em analisar seus conflitos e receber as intervenções da terapeuta.

\section{Discussão}

A análise do processo terapêutico de Valter, descrito acima, com base no CPQ, demonstra características relevantes do tratamento, e sua adesão ao modelo psicanalítico. O uso da interpretação, o trabalho realizado para reduzir as defesas do paciente e para analisar conflitos, juntamente com a atitude exploratória da terapeuta e a ênfase nos estados internos da criança estiveram presentes.

A fase inicial do tratamento foi caracterizada pela busca da terapeuta em familiarizar o paciente com o setting e o processo psicoterápico. Adotou uma postura empática e sensível, incentivando o paciente a falar e procurando estimular sua compreensão sobre seus problemas, buscando estabelcer uma aliança de trabalho (Castro et al., 2009; evidenciado pelos itens 6 - "T é sensível aos sentimentos de C", 31 - "T solicita mais informação ou elaboração" e 2 - "T comenta o comportamento não verbal de C").

Dos três aos nove meses de tratamento, houve um aumento do trabalho interpretativo (conforme itens 36 - "T assinala o uso de defesas por parte de C", 50 - "T chama a atenção para sentimentos considerados inaceitáveis pela criança", 67 - "T interpreta os desejos, sentimentos ou ideias rejeitados ou inconscientes") concomitantemente com a maior expressão de sentimentos negativos, principalmente a agressividade, pela criança (conforme itens 1 - "C expressa sentimentos negativos - ex. crítica, hostilidade - em direção a T", 20 - "C é provocadora; desafia $\mathrm{T}$ ou as regras e limites da sessão", 42 - "C ignora ou rejeita os comentários e observações de T", 58 - "C parece relutante em examinar pensamentos, reações ou motivações relacionadas aos problemas"). A elevação da resistência e ansiedade de Valter também foi muito característico, embora ele tenha demonstrado sentir-se compre- 
endido pela terapeuta (conforme item menos característico 41 - "C não se sente compreendida pelo terapeuta").

A caracterização do processo terapêutico, nesse momento, evidencia uma elevação da ansiedade e resistência de Valter, manifestada por sua relutância em examinar pensamentos, reações ou motivações relacionadas aos problemas, juntamente com suas atitudes hostis e desafiadoras em direção à terapeuta. Embora expressões de desagrado, raiva ou aversão ao terapeuta sejam esperadas e muito importantes ao longo de um tratamento (Calberg, 2009), a externalização desses impulsos, nesse caso, podem significar que, em virtude de apresentar poucos recursos psíquicos, déficits relacionais significativos, capacidade reduzida de insight e de abstração, Valter teve muita dificuldade para reconhecer seus sentimentos, desejos e necessidades e defendeu-se intensamente contra a expressão da influência pulsional (Neubauer, 1996). Nesse sentido, este estudo forneceu uma evidência empírica de que este tratamento demandou a utilização de outras estratégias, que não as prescritas pelo modelo psicanalítico tradicional. Utilizou-se intervenções de apoio, para que o paciente pudesse se sentir mais seguro para explorar o conteúdo de sua própria mente (Goodman et al., 2014).

Um movimento, nesse sentido, foi observado no quinto período analisado, em que as intervenções da terapeuta caracterizaram-se mais por abordagens reflexivas e de apoio do que pelo trabalho interpretativo (conforme os itens: 3 - "Os comentários de T visam encorajar a fala de C", 6 - "T é sensível aos sentimentos da criança", 86 - "T é confiante, seguro de si" 45 - "T tolera os fortes afetos ou impulsos de C" e 98 - "A relação terapêutica é discutida") e Valter, ainda que apresentasse algum nível resistência, revelou maior abertura para o trabalho terapêutico, abordando conteúdos significativos em relação à sua problemática e evidenciando que se sentia compreendido pela terapeuta (conforme o item: 88 - "O material da sessão é significativo e relevante em relação aos conflitos de C" e não característico: 41 - "C não se sente compreendida pelo terapeuta"). As características da interação foram se tornando mais salientes ao longo do tempo e podem significar uma maior aproximação do paciente com a terapeuta, sendo significativo o aparecimento, pela primeira vez, do item referente à discussão da relação terapeutica, que possibilitou a abordagem de temas conflituosos.

Embora não seja possível falar em uma relação causal da mudança no processo terapêutico, é possível que a terapeuta, intuitivamente, percebeu a relação entre as suas intervenções e o aumento dos níveis de ansiedade e resistência de Valter, nos tempos 2, 3 e 4. Consequentemente, empaticamente e gradualmente, começou a modificar suas intervenções para além do que estava prescrito pela sua abordagem teórica. Nesse sentido, passou a apoiar as forças do ego em desenvolvimento da criança, ao invés de trabalhar interpretativamente o acesso aos conteúdos repelidos, oferecendo um enquadre mais reflexivo e de apoio, a fim de promover a construção de representações internas mais estáveis. Este achado sugere que modelos diferentes de tratamento podem funcionar melhor para diferentes tipos de pacientes. Terapeutas emocionalmente sintonizados com seus pacientes podem desenvolver intuitivamente estratégias terapêuticas bem sucedidas, com base nas respostas de seus pacientes, antes que se tornem conscientes dessas estratégias (Goodman et al., 2014).

Esse movimento da terapeuta, possivelmente, provocou algumas mudanças no comportamento de Valter nas sessões, que começou a falar sobre seus conflitos centrais, principalmente no que tange às suas dificuldades relacionais. Fonagy e Target (1996) caracterizam este processo como a necessidade de uma expansão da realidade psíquica através da mentalização ou desenvolvimento da função reflexiva. A mentalização é definida como a habilidade de entender e interpretar implícita e explicitamente o próprio comportamento e o dos outros como expressões de vários estados mentais, como pensamentos, sentimentos, desejos. A psicoterapia pode aprimorar a capacidade de mentalização dos pacientes, por meio de uma postura reflexiva do terapeuta, com intervenções específicas que incluem a representação precisa do estado atual ou passado de sentimentos do paciente e de suas representações internas. Incluem também 
centrar a discussão sobre assuntos diretamente relacionados às crenças, desejos e sentimentos do paciente (Bateman \& Fonagy, 2004).

Algumas pesquisas de processo em psicoterapias têm indicado que, na prática, os psicoterapeutas tendem a usar intervenções de diferentes modalidades (Ablon \& Jones, 1998; Serralta, Pole, Nunes, Eizirik, \& Olsen, 2010). Este uso de recursos diversos para enfrentar os problemas práticos está, por sua vez, relacionado ao que é conhecido como movimento de integração em psicoterapia que consiste em enxergar além das possibilidades de uma única abordagem teórica para ver o que pode ser aprendido de outras perspectivas (Yoshida, 2012).

\section{Considerações Finais}

Este estudo naturalístico oferece uma análise sistemática para o tratamento de uma criança, tal como ele ocorre na prática clínica. Ele difere de projetos experimentais que, emboram permitam inferir causalidade mais fortemente, o fazem à custa de alterar fundamentalmente o processo terapêutico de várias formas (por exemplo, distribuindo aleatoriamente pacientes a tratamentos que eles, de outra forma, poderiam não escolher). Além disso, faz parte da primeira investigação a utilizar o CPQ no contexto brasileiro, para analisar as características do processo psicoterápico com crianças. $\mathrm{O}$ procedimento evidencia como os itens se modificaram ao longo do tempo e como essas modificações podem ser indicativas de evolução do processo terapêutico.

Salienta-se que inúmeros fatores influenciam o processo terapêutico de uma criança. Características de personalidade, conflitos e dificuldades da criança e do terapeuta são elementos presentes no ambiente terapêutico, evidenciando que terapeuta e paciente são autores e coautores do processo psicoterápico. A relação estabelecida entre a díade (incluindo as dimensões de transferência e contratransferência) também representa um fator relevante no processo. Ilustra-se, também, a importante influência das características do paciente sobre as intervenções da terapeuta, o que documenta a importância da colaboração paciente-terapeuta, bem como a in- fluência de estratégias pertencentes a outras modalidades de terapia.

O estudo, também, possui limitações. Trata-se de um estudo de caso único que não pode produzir generalizações sobre os outros casos. Além disso, o número de sessões analisadas (apenas dez) constitui uma importante limitação. Para a maior compreensão do processo psicoterápico, a análise de um maior número de sessões, assim como a utilização de outros meios de análise do material clínico, como medidas de resultado, poderiam agregar novas informações.

Em suma, a análise de psicoterapias com crianças de diferentes idades e quadros clínicos poderá significar não apenas uma contribuição para o avanço teórico deste campo de estudos, mas, também, beneficiar a prática clínica com essa população. Espera-se que isto inspire o crescimento deste tipo de investigação no Brasil.

\section{Referências}

Ablon, J. S., \& Jones, E. E. (1998). How expert clinician's prototypes of an ideal treatment correlate with outcome in psychodynamic and cognitivebehavioral therapy. Psychotherapy Research, $8(1), 71-83$.

Bateman, A., \& Fonagy, P. (2004). Mentalization based treatment of borderline personality disorder. Journal of Personality Disorders, 18, 3651. doi:10.1002/j.2051-5545.2010.tb00255.x

Brum, E. H. M. de, Frizzo, G. B., Gomes, A. G., Silva, M. R., Souza, D. D. de, \& Piccinini, C. A. (2012). Evolução dos modelos de pesquisa em psicoterapia. Estudos de Psicologia (Campinas), 29(2), 259-269. doi:10.1590/S0103$-166 \mathrm{X} 2012000200012$

Calberg, G. (2009). Exploring change processes in psychodynamic child psychotherapy: The terapist's perspective. In N. Midgley, J. Anderson, E. Grainger, T. Vuckovic-Nesic, \& C. Urwin (Eds.), Child psychotherapy and research: New approaches, emerging findings (pp. 100-112). New York: Routledge.

Castro, L. K., Campezatto, P. V. M., \& Saraiva, L. A. (2009). As etapas da psicoterapia com crianças. In M. G. K. Casto \& A. Stürmer (Eds.), Crianças $e$ adolescentes em psicoterapia: Abordagem psicanalítica (pp. 97-115). São Paulo, SP: Artmed. 
Deakin, E. K., \& Nunes, M. L. T. (2008). Investigação em psicoterapia com crianças: Uma revisão. Revista de Psiquiatria do Rio Grande do Sul, 30(1). doi:10.1590/S0101-81082008000200003

Deakin, E. K., \& Nunes, M. L. T. (2009). Effectiveness of child psychoanalytic psychotherapy in a clinical outpatient setting. Journal of Child Psychotherapy, 35(3), 290-301. doi:10.1080/00754170903244621

Edwards, D. J. A. (2007). Collaborative versus adversarial stances in scientific discourse: Implications for the role of systematic case studies in the development of evidence-based practice in psychotherapy. Pragmatic Case Studies in Psychotherapy, 3(1), 6-34.

Eells, T. D. (2007). Generating and generalizing knowledge about psychotherapy from pragmatic case studies. Pragmatic Case Studies in Psychotherapy, 3(1), 35-54.

Efron, A. M., Fainberg, E., Kleiner, Y., Sigal, A. M., \& Woscoboinik, P. (2003). A hora de jogo diagnóstica. In M. L. S. Ocampo, M. E. G. Arzeno, \& E. G. Piccolo (Eds.), O processo psicodiagnóstico e as técnicas projetivas (pp. 207-238). São Paulo, SP: Martins Fontes.

Eizirik, C. L., \& Hauck, S. (2007). Psicanálise e psicoterapia de orientação analítica. In A. V. Cordioli (Ed.), Psicoterapias: Abordagens atuais (3. ed., pp. 151-166). Porto Alegre, RS: Artmed.

Fonagy, P., \& Target, M. (1996). Predictos of outcome in child psychoanalysis: A retrospective study of 763 cases at the Anna Freud Centre. Journal of the American Psychoanalytic Association, 44, 27-77.

Gabbard, G. O., \& Westen, D. (2003). Repensando a ação terapêutica. Revista de Psiquiatria do Rio Grande do Sul, 25(2), 257-273. doi:10.1590/ S0101-81082003000200003

Glenn, J. (1996). Uma visão geral da técnica analítica de crianças. In Psicanálise e psicoterapia de crianças (pp. 7-22). Porto Alegre, RS: Artes Médicas.

Goodman, G., Anderson, K., \& Diener, M. J. (2014). Processes of therapeutic change in psychodynamic therapy of two inpatients with borderline personality disorder. Journal of Psychotherapy Integration, 24(1), 30-45. doi:10.1037/a0035970

Goodman, G., \& Athey-Lloyd, L. (2011). Interaction structures between a child and two therapists in the psychodynamic treatment of a child with
Asperger's disorder. Journal of Child Psychotherapy, 37(3), 311-326. doi:10.1080/007541 7X.2011.614749

Jones, E. E. (1988). Manual for the Psychotherapy Process Q-Set. Unpublished manuscript, University of California at Berkeley, CA.

Jones, E. E. (2000). Therapeutic action, a guide to psychoanalytic therapy. Northvale, NJ: Jason Aronson.

Jones, E. E., Hall, S., \& Parke, L. A. (1991). The process of change: The Berkeley Psychotherapy Research Group. In L. Beutler \& M. Crago (Eds.), Psychotherapy research: An international review of programmatic studies (pp. 98107). Washington, DC: American Psychological Association.

Kazdin, A. E., \& Nock, M. K. (2003). Delineating mechanisms of change in child and adolescent therapy: Methodological issues and research recommendations. Journal of Child Psychology and Psychiatry, 44(8), 1116-1129. doi:10.1111/1469-7610.00195

Luyten, P., Blatt, S. J., \& Mayes, L. C. (2012). Process and outcome in Psychoanalytic Psychotherapy Research: The need for a (relatively) new paradigm. In R. A. Levy, J. S. Ablon, H. Kächele (Eds.), J. F. Rosenbaum, \& S. Cobb (Series Ed.), Psychodynamic Psychotherapy Research: Evidence-based pratice and praticebased evidence: Current Clinical Psychiatry (pp. 346-359). doi:10.1007/978-1-60761-792-1

Midgley, N. (2007). Researching the process of psychoanalytic child psychotherapy. In E. Kennedy \& N. Midgley, Process and outcome research in child, adolescent and parent-infant psychotherapy: A thematic review (pp. 8-53). London: North Central London Strategic Health Authority.

Midgley, N., Anderson, J., Grainger, E., VuckovicNesic, T., \& Urwin, C. (Eds.). (2009). Child psychotherapy and research: New approaches, emerging findings. New York: Routledge.

Midgley, N., \& Kennedy, E. (2011). Psychodynamic psychotherapy for children and adolescents: A critical review of the evidence base. Journal of Child Psychotherapy, 37(3), 1-29. doi:10.1080/ 0075417X.2011.614738

Neubauer, P. (1996). A fase inicial da análise de crianças. In J. Glenn (Ed.), Psicanálise e psicoterapia de crianças (pp. 161-168). Porto Alegre, RS: Artes Médicas. 
Odhammar, F., Sundin, E. C., Jonson, M., \& Carlberg, G. (2011). Children in psychodynamic psychotherapy: Changes in global functioning. Journal of Child Psychotherapy, 37(3), 261279. doi:10.1080/0075417X.2011.614744

Oliveira, I. T. (2012). Psicoterapia breve psicodinâmica de crianças. In M. E. N. Lipp \& E. M. P. Yoshida, Psicoterapia breve nos diferentes estágios evolutivos. São Paulo, SP: Casa do Psicólogo.

Palmer, R., Nascimento, L. N., \& Fonagy, P. (2013). The state of the evidence base for psychodynamic psychotherapy for children and adolescents. Child \& Adolescent Psychiatric Clinics of North America, 22(2), 149-214. doi:10.1016/j. chc.2012.12.001

Ramires, V. R. R., \& Schneider, C. (no prelo). Psicoterapia de Crianças: Desenvolvimento da versão em português do Child Psychotherapy Q-Set. Psicologia: Teoria e Pesquisa.

Schneider, C. (2003). The development of the Child Psychotherapy Q-set (Doctoral dissertation). University of California, Berkeley, CA.

Schneider, C. (2004). 'The development of the Child Psychotherapy Q-Set'. Dissertation Abstracts International: Section B. Sciences and Engineering, 65(2-B), 1039.

Schneider, C., \& Jones, E. E. (2006). Child Psychotherapy $Q$-Set. Coding Manual. Unpublished manuscript, University of California, Berkeley, CA.

Schneider, C., \& Jones, E. E. (2012). Appendix IB. Child Psychotherapy Q-Set. Coding Manual. In R. A. Levy, J. S. Ablon, \& H. Kächele (Eds.), Psychodynamic Psychotherapy Research: Evidence-based practice and practice-based evidence (pp. 611-626). New York: Humana Press.

Schneider, C., Midgley, N., \& Duncan, A. (2010). A "motion portrait" of a psychodynamic treatment of an 11-year-old girl: Exploring interrelations of psychotherapy process and outcome using the Child Psychotherapy Q-Set. Journal of Infant, Child and Adolescent Psychotherapy, 9, 98-111. doi:10.1080/15289168.2010.510979
Schneider, C., Pruetzel-Thomas, A., \& Midgley, N. (2009). Discovering new ways of seeing and speaking about psychotherapy process: The child psychotherapy Q-set. In N. Midgley (Ed.), Child Psychotherapy and research (pp. 72-84). New York: Taylor \& Francis

Serralta, F. B., Nunes, M. L., \& Eizirik, C. (2007). Elaboração da versão em português do Psychotherapy Process Q-Set. Revista de Psiquiatria do Rio Grande do Sul, 29(1), 44-55. doi:10.1590/ S0101-81082007000100011

Serralta, F. B., Pole, N., Nunes, M. L., Eizirik, C., \& Olsen, C. (2010). The process of change in brief psychotherapy: Effects of psychodynamic and cognitive-behavioral prototypes. Psychotherapy Research, 20(5), 564-575. doi:10.1080/1050330 7.2010 .493537

Strupp, H. H. (2013). The outcome problem in psychotherapy revisited. American Psychological Association, 50(1), 3-11. doi:10.1037/ h0094491

Yoshida, E. M. P. (2012). Desenvolvimentos futuros das Psicoterapias Breves. In M. E. N. Lipp \& E. M. P. Yoshida, Psicoterapia breve nos diferentes estágios evolutivos. São Paulo, SP: Casa do Psicólogo.

Zavaschi, M. L., Bassols, A. M., Bergmann, D. S., \& Costa, F. M. (2005). Abordagem psicodinâmica na infância. In C. Eizirik, R. Aguiar, \& S. Schestatsky, Psicoterapia de orientação analitica-Fundamentos teóricos e clínicos (2. ed., pp. 717-737). Porto Alegre, RS: Artmed.
Recebido: 19/02/2015

$1^{a}$ revisão: 04/08/2015

$2^{a}$ revisão: $1 \% 10 / 2015$ Aceite final: $1 \% 10 / 2015$ 